\title{
Analyse tridimensionnelle du comportement des tunnels dans des terrains meubles
}

\section{SHAHROUR}

S. GHORBANBEIGI

Laboratoire de Mécanique de Lille (URA 1441)

École Centrale de Lille, 59651 Villeneuve-d'Ascq
Dans cet article on présente une analyse du

comportement tridimensionnel des tunnels peu profonds. L'analyse est effectuée à l'aide d'un programme d'éléments finis (PEC3D) qui a été spécifiquement développé pour le calcul tridimensionnel des tunnels construits dans des terrains meubles. Après une analyse du comportement d'un tunnel dans un terrain argileux, on présente une étude de l'influence sur ce comportement des paramètres significatifs, tels que l'indice de stabilité, la hauteur de recouvrement, les contraintes initiales et l'épaisseur des tranches d'excavation. Les résultats des simulations tridimensionnelles seront utilisés pour étudier la possibilité d'appliquer la méthode de convergenceconfinement au calcul des tunnels peu profonds.

\section{Analysis of the three dimensional behaviour of tunnels in soft soils}

This paper contains a three dimensional analysis of the behaviour of tunneis in soft ground. This analysis is carried out using a finite element program (PEC3D) which was specifically developed for the calculation of shallow tunnels. After a study of the behaviour of a tunnel in a clayey soil, we propose to analyse the influence on this behaviour of significant parameters such as : the stability ratio, the cover thickness, the initial stresses and the width of the excavated bloc. Finally, results of the three dimensional analysis are used to study the use of the convergence - confinement method in the calculation of shallow tunnels. 


\section{Introduction}

Des observations in situ et des analyses théoriques montrent que le champ de déplacement au voisinage du front de taille est tridimensionnel (Ranken et Ghaboussi, 1975. Wittke et Gell, 1980), Lo et al., 1984, Leca et Clough, 1989). Ces mouvements peuvent être très importants si le front de taille n'est pas suffisamment stable ou si le tunnel a une forte interaction avec des ouvrages existants. Dans ce cas, l'analyse bidimensionnelle n'est pas satisfaisante, et on doit avoir recours à un calcul tridimensionnel qui prend en compte des aspects particuliers tels que le comportement non linéaire des sols, les phases de construction, l'interaction sol-revêtement et les conditions hydrauliques du terrain. Vu la complexité et le coùt de ce type d'analyses, des travaux de modélisation sont généralement effectués avec des hypothèses simplifiées au niveau du comportement du sol et des phases de construction ou en utilisant des moyens informatiques puissants (Wittke et Gell, 1980, Kasali et Glough, 1982, Swoboda et al., 1989, Lee et Rowe, 1990).

Afin de rendre plus aisé le calcul tridimensionnel des tunnels, le Laboratoire de Mécanique de Lille a développé un programme spécifique qui permet, grâce à une méthode de stockage particulière de la matrice de rigidité où seuls les termes non nuls sont pris en compte, de réaliser des calculs tridimensionnels et non linéaires sur des stations de travail de capacité moyenne et dans des temps raisonnables. Des outils ont été également construits pour la génération automatique de l'avancement du front de taille et l'application d'une pression de confinement à ce front. Une description détaillée de ce programme a été donnée par Shahrour et Ghorbanbeigi (1993 et 1994).

Dans cet article, on propose d'appliquer ce programme à l'analyse du comportement tridimensionnel d'un tunnel dans un terrain argileux et d'étudier l'influence sur ce comportement de principaux paramètres (indice de stabilité, profondeur, contraintes in situ et épaisseur d'excavation), Les résultats des simulations tridimensionnelles seront utilisés pour étudier la possibilité d'appliquer la méthode de convergenceconfinement au calcul des tunnels peu profonds.

\section{2.}

\section{Analyse du comportement d'un tunnel dans un sol argileux}

\section{1}

\section{Présentation de l'ouvrage de référence}

L'exemple retenu dans ce travail s'inspire d'une étude présentée par Lee et Rowe (1990). Il s'agit d'un tunnel à section circulaire non revêtu avec un diamètre $\mathrm{D}=5 \mathrm{~m}$, creusé dans un sol argileux de $20 \mathrm{~m}$ d'épaisseur reposant sur un substratum rigide. Le tunnel est centré sur un axe horizontal situé à une profondeur de 2D. Le rapport entre la hauteur de couverture (la distance entre le centre du tunnel et la surface) et le diamètre $(H / D)$ est égal à 2.

L'analyse a été effectuée à court terme (condition non drainée) en supposant que le comportement du sol est élastique parfaitement plastique obéissant au critère de Tresca. Le sol est composé de deux couches. La première couche a une épaisseur de $2,5 \mathrm{~m}$ avec une cohésion constante $\mathrm{C}_{4}=20 \mathrm{kPa}$. Dans la deuxième couche, la cohésion augmente linéairement avec la profondeur (z):

$$
\mathrm{C}_{\mathrm{u}}=9,2 \mathrm{z}-3 \mathrm{C}_{\mathrm{u}}>20 \mathrm{kPa}
$$

Le module d'Young a été supposé proportionnel à la cohésion non drainée selon la relation: $E=200 C_{u}$. Le coefficient de Poisson a été supposé constant dans tout le massif $v=0,49$. La distribution des contraintes initiales a été supposée de type cdométrique avec un poids volumique $\gamma=20 \mathrm{kN} / \mathrm{m}^{3}$ et un coefficient de pression des terres au repos $\mathrm{Ko}=1$.

Pour les conditions aux limites, on a supposé que les déplacements horizontaux parallèles à l'axe du tunnel sont bloqués à $25 \mathrm{~m}$ (5D) à l'avant et à l'arrière du front de taille. Les déplacements horizontaux dans le plan perpendiculaire à l'axe du tunnel sont aussi bloqués à $25 \mathrm{~m}$ de l'axe du tunnel. Les déplacements verticaux sont bloqués au niveau du substratum rigide. Le chargement dû à l'excavation a été appliqué en plusieurs incréments.

Le maillage utilisé est montré dans la figure 1. Il comprend 3024 éléments Q16 et 10825 nceuds. Il est raffiné au voisinage de l'axe du tunnel et du front de taille pour une prise en compte de la variation des contraintes dans ces zones. Le nombre de degrés de liberté est de 29434. La place mémoire nécessaire pour le stockage de la matrice de rigidité avec la méthode retenue dans notre programme est de l'ordre de $4 \mathrm{Mo}$ soit de l'ordre de $10 \%$ de celle nécessaire pour un stockage en lignes de ciel.

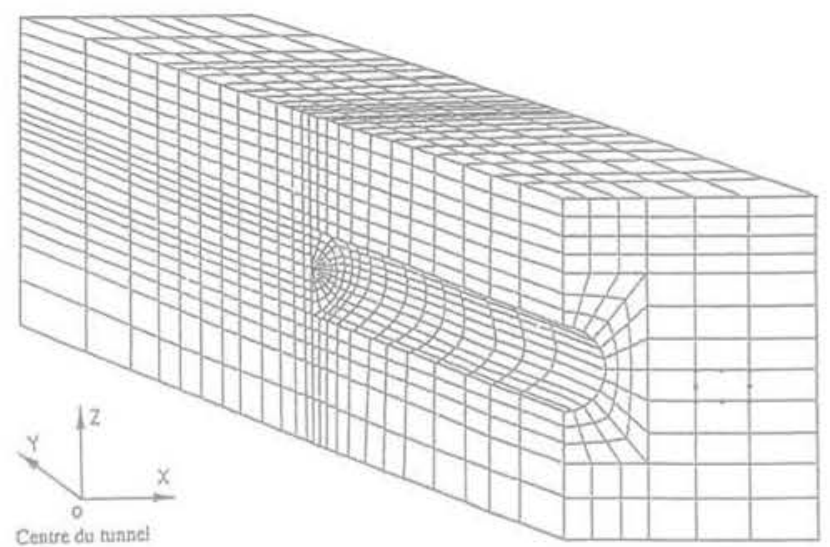

FG. I Maillage utilisé - 3024 éléments et 10825 nceuds.

Finite element mesh -3024 elements and 10825 nodes

\section{2}

\section{Analyse des résultats de calcul}

Les figures $2 \mathrm{a}$ et $2 \mathrm{~b}$ montrent respectivement les déformées d'une section transversale à 3 diamètres à l'arrière du front de taille $(\mathrm{Y}=-3 \mathrm{D})$ et d'une section longitudinale dans le plan de symétrie $(X=0)$. On constate que le champ de déplacement est orienté vers l'axe du tunnel. A la surface du massif du sol on note un tassement en forme de cuvette. Dans la section transversale, le déplacement augmente en s'approchant de l'axe du tunnel. Dans la section longitudinale, on note que le déplacement augmente en s'éloignant du front de taille 


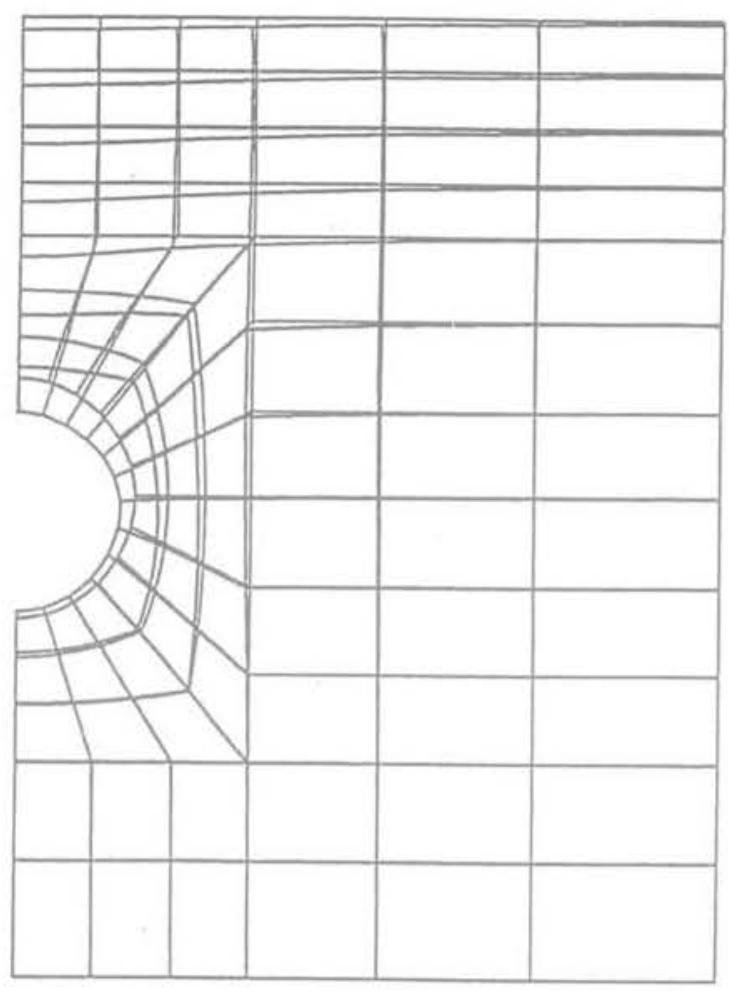

et devient de type plan à une distance de l'ordre de 1,5 D du front de taille.

La figure 3 montre l'évolution du déplacement vertical dans le plan de symétrie $(X=0)$ pour différentes profondeurs. On note que ce déplacement est très faible à l'avant du front de taille, mais il augmente rapidement au passage de ce front et tend à se stabiliser à partir d'une distance du front de taille variant entre une à trois fois le diamètre. Au niveau du radier, on note un soulèvement du sol qui se stabilise rapidement au passage du front de taille (à une distance de l'ordre du diamètre de celui-ci). Au niveau de la clé, on note un tassement important qui évolue avec la distance du front de taille et tend à se stabiliser à partir d'une distance de l'ordre de trois diamètres du front de taille. Le tassement maximal atteint en clé est de l'ordre de 0,028D. A la surface du massif, on note un tassement inférieur à celui obtenu au niveau de la clé. Ce tassement tend à se stabiliser à partir d'une distance de l'ordre de 3D du front de taille avec un tassement maximal de l'ordre de $0,01 \mathrm{D}$.

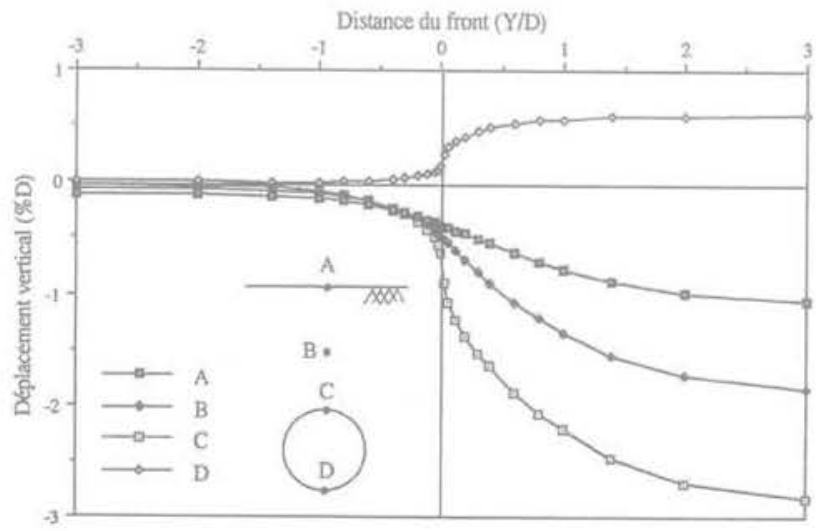

FIG. 3 Déplacement vertical dans le plan de symétrie $(X=0)$.

Vertical displacement in the longitudinal plane of symmetry $(X=0)$.

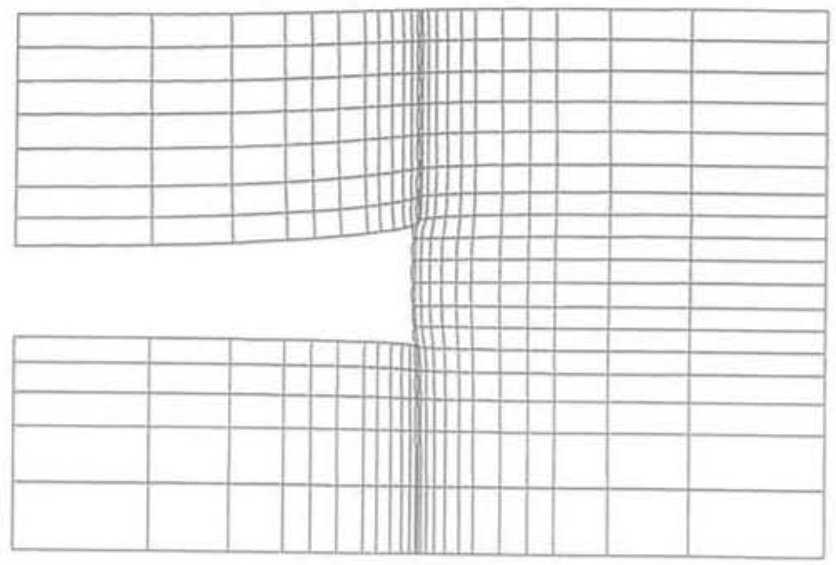

HG. 2 Déformée du tunnel:

a) section tansversale à 3 diamètres à l'arrière du front de taille $(\mathrm{Y}=3 \mathrm{D})$;

b) section longitudinale dans le plan de symétrie $(X=0)$.

Deformation of the tunnel:

a) transverse section at three diameters behind the tunnel face $(Y=3 D)$;

b) longitudinal plane of symmetry $(X=0)$.

La figure 4 illustre le profil du déplacement axial dans la section du front de taille et dans le plan de symétrie. On constate que ce déplacement est faible en dehors de la zone d'excavation. Le déplacement du front de taille est relativement uniforme et atteint son maximum (de l'ordre de 0,007D) vers le centre du front.

Les champs de déplacement radial autour de la périphérie du tunnel dans la section du front de taille et dans une section à 3 diamètres à l'arrière du front sont montrés dans les figures $5 \mathrm{a}$ et $5 \mathrm{~b}$. On constate que l'amplitude du déplacement diminue de manière continue en partant de la clé de voûte vers le radier, et qu'il est plus important en arrière du front de taille.

La figure 6 montre l'évolution du déplacement horizontal $\left(\mathrm{u}_{\mathrm{x}}\right)$ à un diamètre de l'axe de symétrie $(\mathrm{X}=1 \mathrm{D})$. On constate que le mouvement horizontal commence à avoir lieu à partir d'une distance de l'ordre d'un diamètre devant le front de taille. Ce déplacement augmente graduellement en s'approchant de ce front et puis rapidement après son passage. En ce qui concerne sa variation avec la profondeur, on note une augmen-

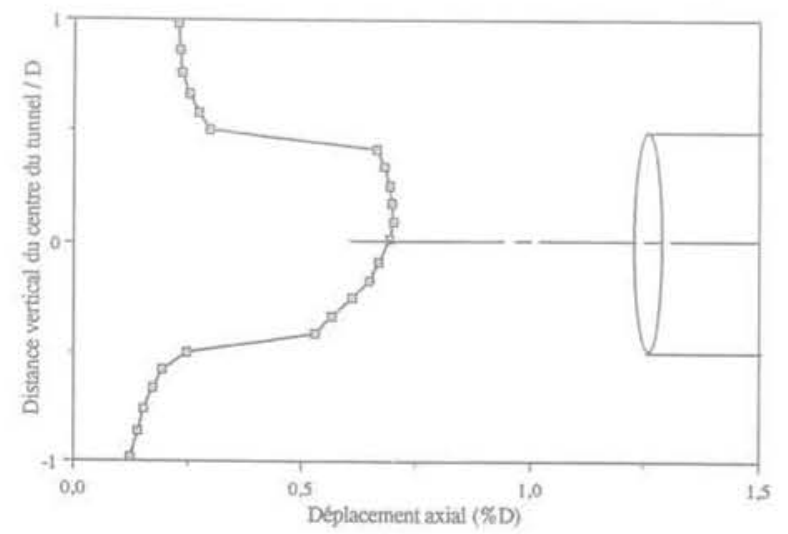

FIG.4 Déplacement axial au front de taille dans le plan de symétrie.

Axial displacement at the tunnel face in the plane of symmetry $(Y=0, X=0)$. 

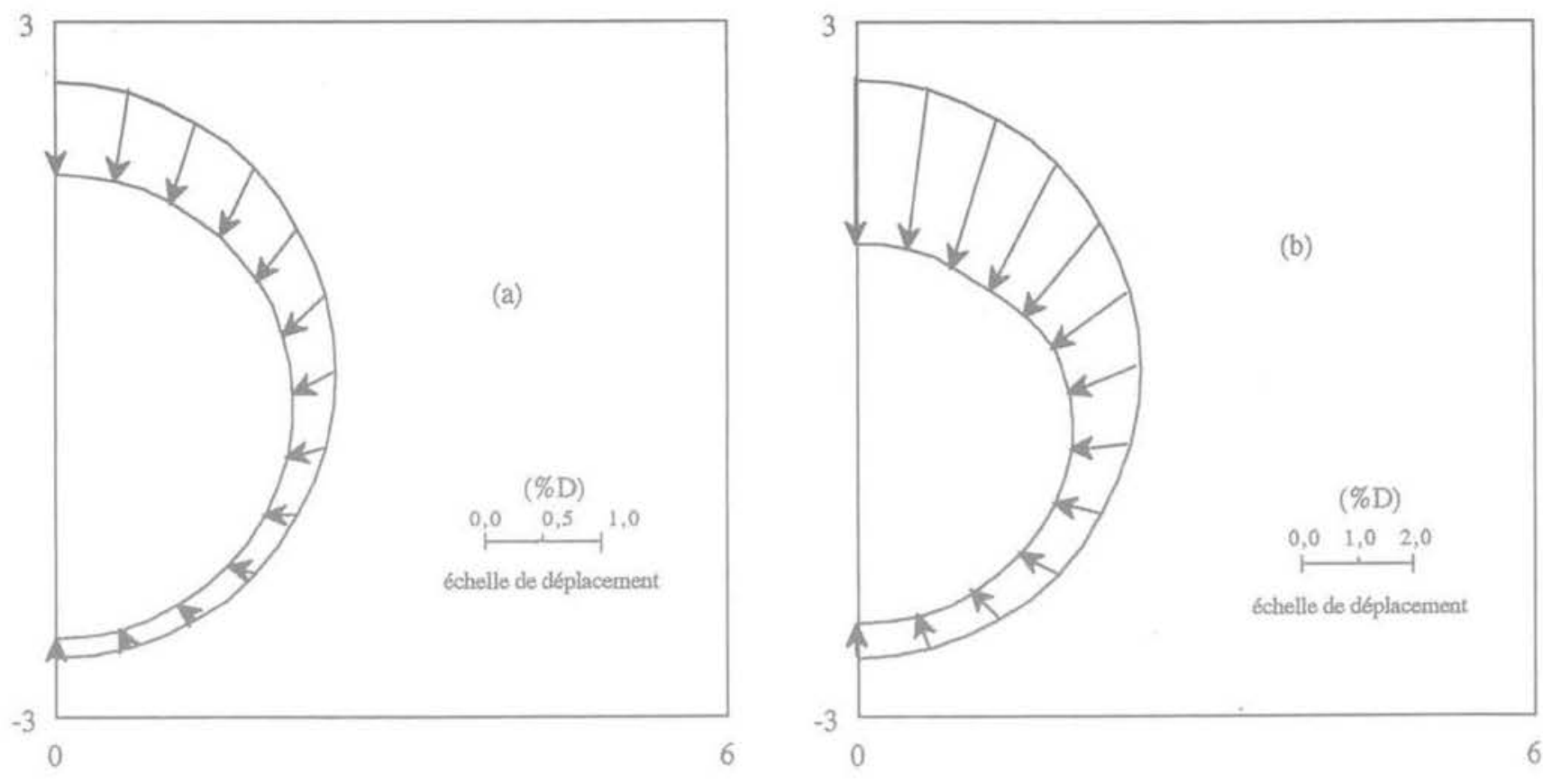

FIG,s Déplacement radial à la périphérie du tunnel

a) au front de taille $(\mathrm{Y}=0)$;

b) à 3 diamètres à l'arrière du front de taille $(\mathrm{Y}=3 \mathrm{D})$.

Radial displacement around the tunnel

a) at the turnel face $(\mathrm{Y}=0)$;

b) at three diameters behind the tunnel face $(\mathrm{Y}=3 \mathrm{D})$.

tation jusqu'au niveau de l'axe du tunnel, suivie par une diminution. L'amplitude du déplacement maximal atteint est de l'ordre de 0,005D.

La figure 7 montre le tassement induit en surface par le creusement. On constate que le tassement augmente en s'approchant de l'axe du tunnel. Le tassement maximal dans la section du front de taille est de l'ordre de $0,004 \mathrm{D}$. Ce déplacement diminue en avant du front, et se réduit à $0,002 \mathrm{D}$ à une distance de l'ordre d'un diamètre du front. Au passage du front de taille, le profil des tassements en surface passe d'un profil relativement plat à un profil évasé.

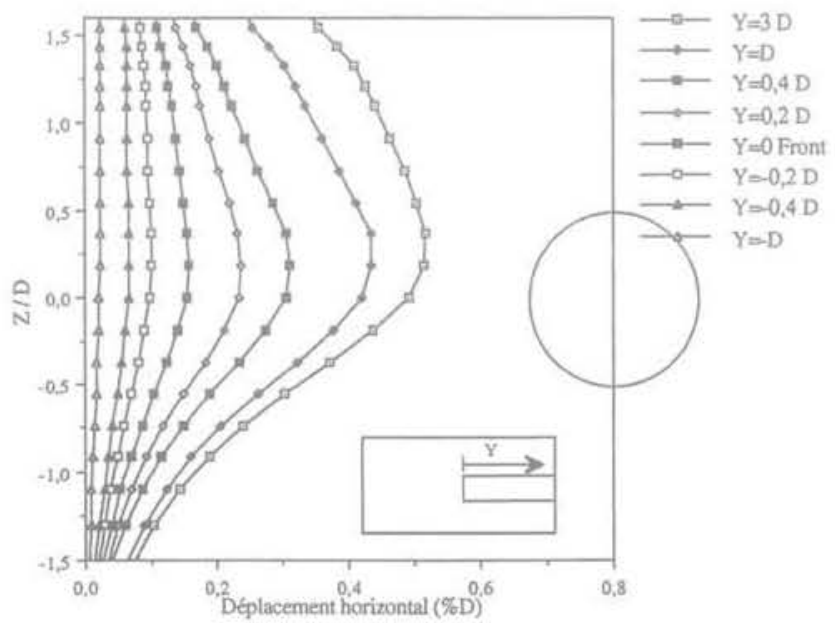

FIG.6 Déplacement horizontal à un diamètre de l'axe du tunnel $(X=1 D)$.

Horizontal displacement at one diameter from the tunnel axis $(X=1 D)$.

\section{Utilisation de la méthode convergence-confinement}

Pour les tunnels profonds, l'effet tridimensionnel peut être pris en compte en utilisant la méthode de convergence-confinement (Panet et Guellec, 1974). Dans cette méthode, l'avancement du tunnel est modélisé par une réduction de la pression radiale interne $\left(\sigma_{f}\right)$ selon l'expression:

$$
\sigma_{r}=\sigma_{r 0}(1-\lambda)
$$

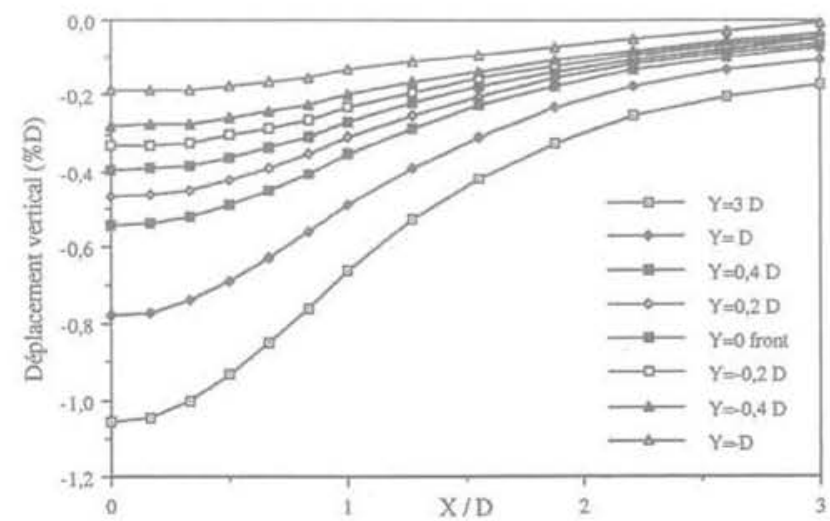

FiG.7 Tassements induits en surface. Settlement induced at the ground surface. 
$\sigma_{\mathrm{r} 0}$ est la pression initiale, $\lambda$ est le taux de déconfinement qui dépend de la distance du front de taille et des propriétés du sol.

L'application de cette méthode aux tunnels peu profonds se heurte à une difficulté majeure celle de la non uniformité du champ de contrainte à la périphérie du tunnel. Dans cette section, nous allons examiner la possibilité d'utiliser cette méthode en travaillant avec le champ de contraintes initiales dans le sol. Le déconfinement sera appliqué selon l'expression : $\sigma=\sigma_{0}(1-\lambda)$ ou $\sigma_{0}$ représente le tenseur de contraintes initiales dans le massif du sol.

Un calcul plan a été effectué avec un taux de déconfinement variant entre 0 et 1. L'évolution des déplacements en quelques points autour de la galerie en fonction de $\lambda$ est illustrée dans la figure 8 . On peut constater que jusqu'à $\lambda=0,6$, l'évolution des déplacements est presque linéaire ensuite elle devient non linéaire. Pour tester la méthode convergence-confinement, nous avons cherché à caler les résultats du calcul plan à ceux du calcul tridimensionnel en utilisant l'expression suivante pour le taux de déconfinement (Panet et Guénot, 1982):

$$
\lambda=\lambda o+\left(1-\left(\frac{a}{a+y}\right)^{2}\right)(1-\lambda o) \quad y=\frac{Y}{D}
$$

où $\mathrm{Y}$ et $\mathrm{D}$ désignent respectivement la distance du front de taille et le diamètre du tunnel. Les deux paramètres $\lambda_{\mathrm{o}}$ et a dpendent des caractéristiques du tunnel. Une procédure de calage nous a permis de déterminer les valeurs de ces paramètres $\left(\mathrm{a}=0,45\right.$ et $\left.\lambda_{\mathrm{f}}=0,4\right)$. La figure 9 montre une confrontation des résultats du calcul tridimensionnel à ceux obtenus avec la méthode de déconfinement. On note un bon accord entre ces résultats.

\section{Étude paramétrique}

Dans cette partie, on présente une étude de linfluence sur le comportement du tunnel présenté cidessus de quatre facteurs importants: l'indice de stabilité, la hauteur de recouvrement, les contraintes initiales et l'épaisseur des tranches d'excavation.

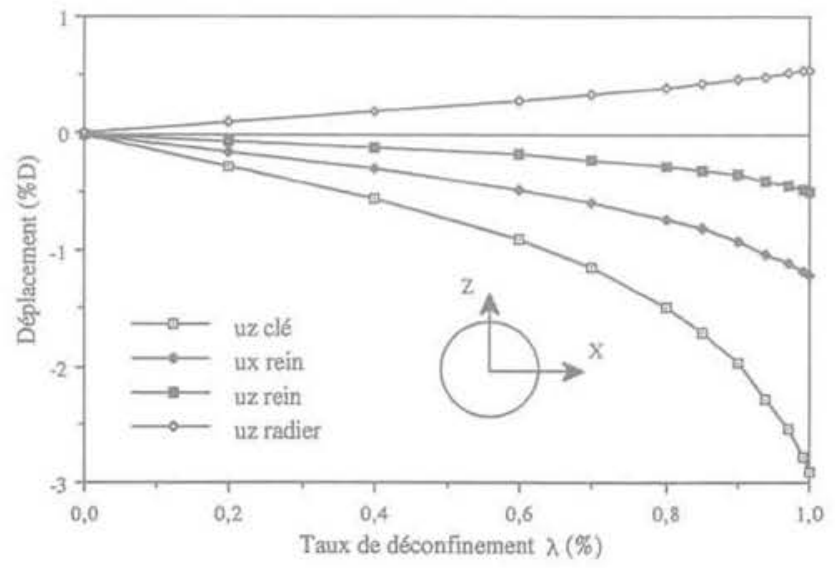

FG. 8 Déplacements obtenus avec la méthode convergence-confinement (calcul plan). Displacement obtained with the convergenceconfinement method (2D analysis).

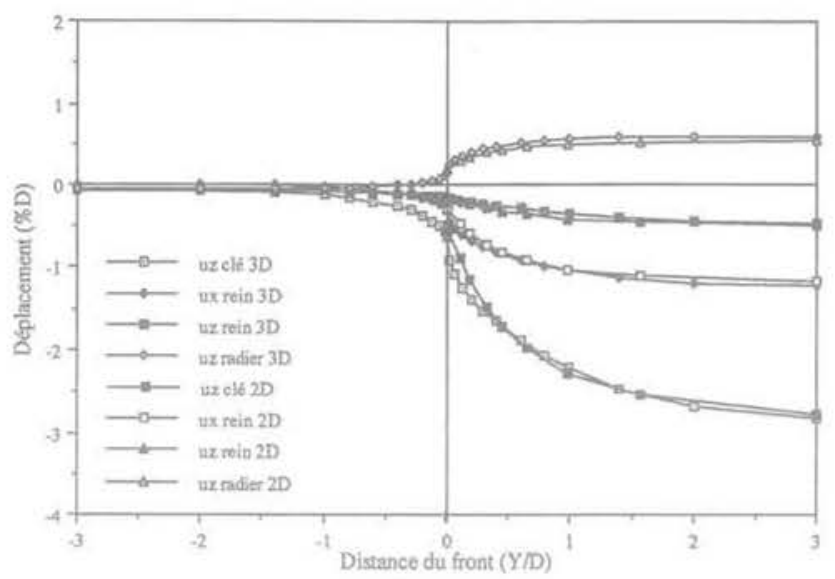

FG.9 Calage du taux de déconfinement sur les résultats de calcul tridimensionnel. Fitting of the deconfinement parameter on the three dimensional analysis.

\section{Indice de stabilité}

L'indice de stabilité permet de situer le niveau de chargement induit par le creusement d'un tunnel dans un sol argileux par rapport à la résistance de ce sol à court terme (Broms et Bennermark, 1967). Il est donné par l'expression: $\mathrm{N}=\frac{\left(\mathrm{P}_{\mathrm{v}}-\mathrm{P}_{\mathrm{f}}\right)}{\mathrm{C}_{u}}$ ou $\mathrm{P}_{\mathrm{v}}$ est la contrainte

verticale, $\mathrm{P}$ est la suppression éventuelle à l'intérieur du tunnel et $\mathrm{C}_{u}$ est la cohésion non drainée. Cet indice constitue, pour un calcul en déformations des tunnels peu profonds, une bonne indication du niveau de plasticité induit par le creusement.

Afin d'étudier l'effet du niveau de plasticité sur le champ de déplacement induit par le creusement du tunnel, nous avons effectué des calculs avec différentes valeurs de l'indice de stabilité ( $\mathrm{N}=1 ; 2 ; 2,25$ et 2,5$)$ correspondant à différentes valeurs de la cohésion non drainée. Les résultats obtenus sont illustrés dans la figure 10. Sur cette figure, nous avons également reporté les déplacements obtenus par une analyse en déformation plane (D.P.). On peut observer que le niveau de plasticité a un effet très important sur l'amplitude et la forme du déplacement en arrière du front. Pour une analyse élastique $(\mathrm{N}=1)$, les déplacements calculés par une analyse tridimensionnelle et par une analyse en déformations planes sont approximativement identiques, ce qui indique que les conditions de déformations planes sont vérifiées à une distance de l'ordre d'un à deux diamètres à l'arrière du front de taille. Mais quand l'indice de stabilité augmente, l'écart entre les déplacements calculés par une analyse tridimensionnelle et par une analyse en déformations planes augmente. Ceci montre qu'en augmentant l'indice de stabilité, les conditions de déformations planes ne sont vérifiées qu'à une distance plus éloignée du front de taille. On peut donc conclure que la distance en arrière du front où les conditions de déformations planes sont atteintes dépend du degré de plasticité développé autour du tunnel. En avant du front de taille, on note également une amplification du déplacement vertical avec la valeur de l'indice de stabilité $\mathrm{N}$.

Le rapport entre le déplacement vertical en clé de voûte au front de taille et le déplacement maximal cal- 


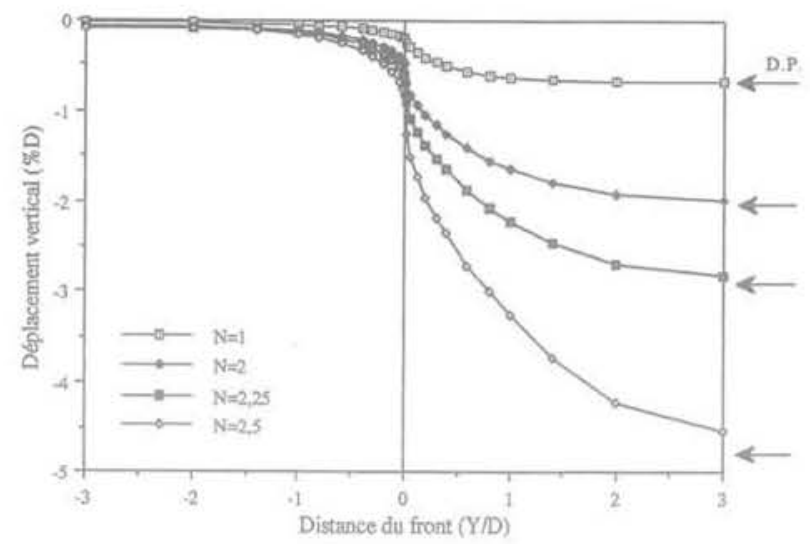

คG. 10 Influence de l'indice de stabilité sur les déplacements le long de la clé de voûte. Influence of the stability ratio on the displacement along the tunnel crown.

culé par une analyse en déformations planes peut être utilisé comme un paramètre pour représenter les mouvements radiaux tridimensionnels qui ont lieu à l'avant du front. Pour ce rapport, plusieurs auteurs ont proposé une valeur de l'ordre de $1 / 3$ pour un milieu élastique (Lo et al., 1984, Lee et Rowe, 1990). Nous avons obtenu une valeur de l'ordre de 0,3 dans le calcul élastique $(\mathrm{N}=1)$ qui est en bon accord avec les valeurs proposées. Cependant, nous avons noté une réduction de ce rapport avec la plasticité. En effet ce rapport n'est que de 0,23 pour $\mathrm{N}=2,5$.

\section{2}

\section{Hauteur de recouvrement}

Des calculs ont été effectués pour trois valeurs du recouvrement (rapport $\mathrm{H} / \mathrm{D}=2 ; 2,5$ et 3 ). Les figures 11a et $11 \mathrm{~b}$ montrent l'influence du rapport H/D sur les déplacements verticaux en clé de voûte et en surface. On constate que ce rapport influence fortement les déplacements induits par le creusement à l'arrière du front de taille. Le déplacement vertical en clé pour $H / D=2$ est $40 \%$ (resp. $60 \%$ ) plus grand que celui obtenu par $\mathrm{H} / \mathrm{D}=2,5$ (resp. $\mathrm{H} / \mathrm{D}=3$ ). L'influence de ce rapport sur les déplacements verticaux à la surface est également très importante. En effet, le déplacement maximal à la surface pour $\mathrm{H} / \mathrm{D}=2$ est 2,2 (resp. 1,6) fois celui obtenu avec $\mathrm{H} / \mathrm{D}=2,5$ (resp. $\mathrm{H} / \mathrm{D}=3$ ).
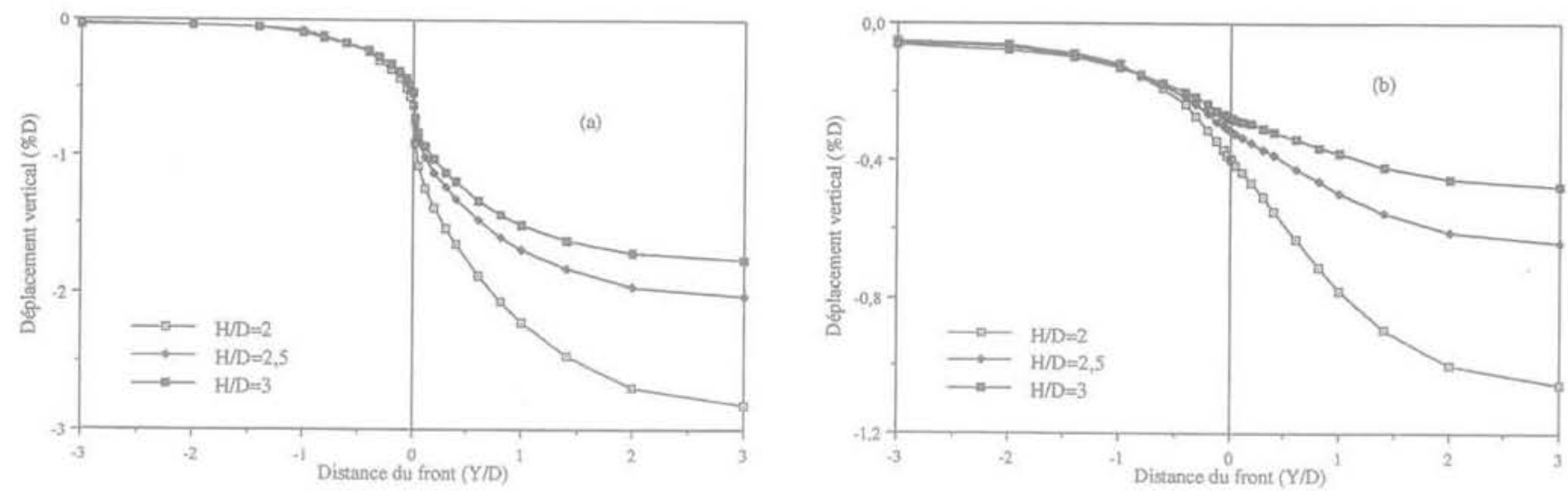

Fig. 11 Influence de la hauteur de recouvrement sur le déplacement vertical le long de l'axe du tunnel a) en clé de voûte;

b) à la surface.

Influence of the cover thickness on the vertical displacement.

a) at the crown

b) at the ground surface.
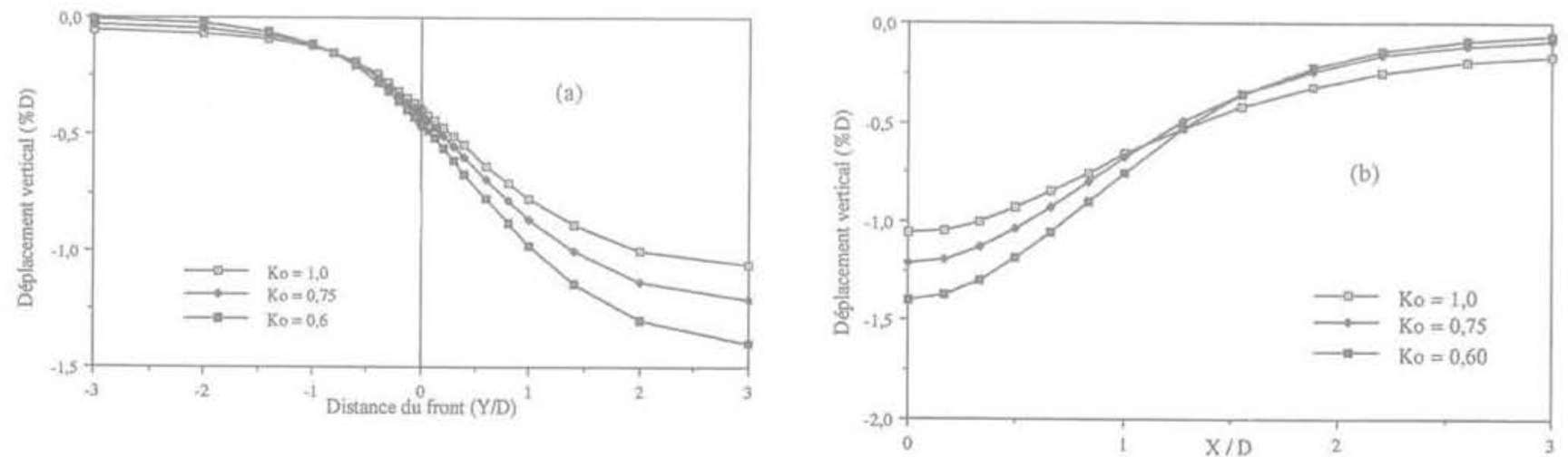

FIG. 12 Influence de l'état initial sur les tassements en surface

a) le long de l'axe du tunnel;

b) à 3 diamètres en arrière du front.

Influence of the initial stresses on the soil settlement

a) along the funnel axis :

b) at three diameters behind the tunnel face: 


\section{Contraintes initiales}

Les figures $12 \mathrm{a}$ et $12 \mathrm{~b}$ montrent l'influence du coefficient de poussée des terres au repos (Ko) sur les tassements en surface. On constate que la diminution de ce coefficient, qui correspond à une augmentation de l'anisotropie des contraintes initiales, a pour effet d'augmenter les tassements à l'arrière du front de taille et de les réduire à l'avant du front. Le premier effet est dû à une amplification de la plasticité alors que le second peut être attribué à la réduction de la décompression des contraintes latérales.

\section{4}

\section{Épaisseur des tranches d'excavation}

Pour étudier l'effet de l'épaisseur des tranches d'excavation retenue dans les simulations numériques, deux séries de calculs ont été effectuées. Dans la première, l'excavation a été modélisée par des tranches dont l'épaisseur est égale à $0,6 \mathrm{D}$; dans la deuxième, les simulations ont été effectuées avec une excavation instantanée. Les résultats obtenus avec deux indices de stabilité sont donnés dans la figure 13. On constate que l'excavation par tranches de 0,6D induit un déplacement plus important que celui obtenu par une excavation instantanée et que l'écart entre les deux déplacements s'amplifie avec l'indice de stabilité. Cet écart est attribué au fait qu'une excavation par des tranches de faible épaisseur permet une bonne extension des déformations plastiques dans le massif et par conséquent une augmentation des tassements. Pour l'exemple traité, l'excavation par tranche de $0,6 \mathrm{D}$ donne un déplacement en clé de voûte qui est supérieur de $5 \%$ (resp. $15 \%$ ) à celui obtenu par une excavation en une seule étape avec un indice de stabilité de 2,25 (resp. 2,5).

\section{4}

\section{Conclusion}

Dans cet article nous avons présenté une analyse du comportement tridimensionnel d'un tunnel peu pro-

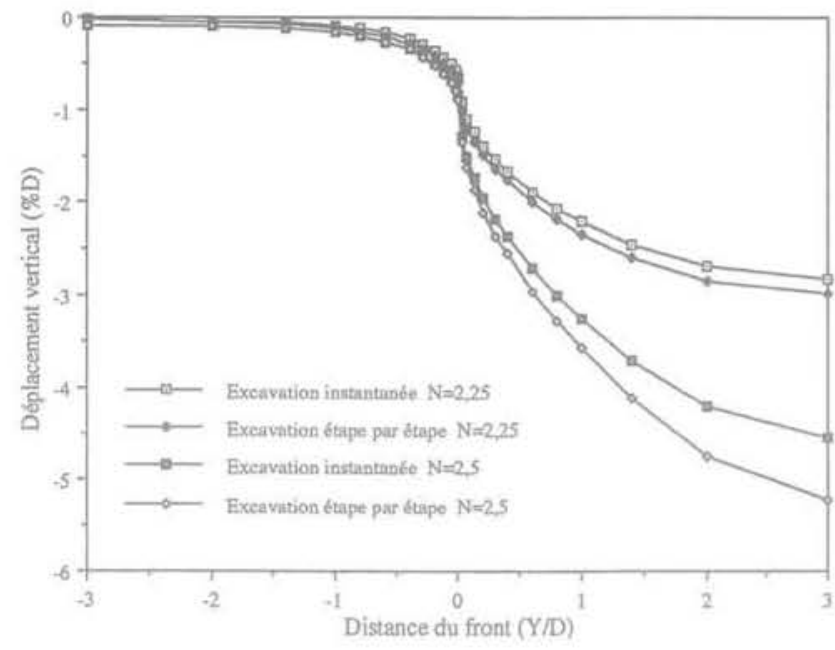

FIG. 13 Influence de l'épaisseur des tranches d'excavation sur le déplacement en clé de voûte.

Influence of the width of the excavated bloc on the displacement at the tunnel crown.

fond construit dans un sol argileux à l'aide du programme de calcul par éléments finis (PEC3D) qui a été spécifiquement développé pour le calcul tridimensionnel des tunnels construits dans des terrains meubles. Les résultats de calcul ont montré le caractère tridimensionnel du champ de déplacement dans une zone située au voisinage du front de taille. L'extension de cette zone et l'amplitude du champ de déplacement dépendent de plusieurs paramètres, notamment l'indice de stabilité, la hauteur de recouvrement et les contraintes initiales.

Une confrontation des résultats de calcul tridimensionnel et d'un calcul plan mené avec la méthode de déconfinement a permis de caler une expression simple pour l'évolution du taux de déconfinement en fonction de la distance du front de taille. Les résultats de calage sont très encourageants et permettent d'envisager l'application de la méthode de convergence-confinement aux tunnels peu profonds.

\section{Bibliographie}

Broms B.B., Bennermark H. - a Stability of clay at vertical openings $x$, Journal of ASCE vol. 93, SMI, 1967, p. 71-94.

Kasali G., Clough W. - $\alpha$ Three dimensional finite element analysis - Advanced and conventional shield tunnelling $x$, Development of a design technology for ground support for tunnels in soil, vol. II. US Department of Transportation, 1982.

Leca E., Clough G.W. - «Modélisation et méthode de dimensionnement, utilisation de la méthode des éléments finis pour l'analyse en terrain meubles. Tunnels et microtunnels en terrain meuble, du chantier à la théorie, Actes du colloque Tunnels et Microtunnels en Terrain Meuble, Presses de I'ENPC, 1989, p. $531-573$.

Lee K.M., Rowe R.K. - « Finite element modelling of the 3-dimensional ground deformation due to tunneling in soft cohesive soils 》, Computers and Geotechnics, vol. 2, 1990, p. 87-138.
Lo K.X., Ng R.M.C., Rowe R.K. - «Predicting settlement due to tunnelling in clays m. Tunneling in soil and rock, ASCE Geotech. III Conference, Atlanta, Georgia, 1984, p. 48-76.

Panet M. Guellec P. - " Contribution à l'étude du soutènement d'un tunne! à larrière du front de taille w. Proc. 3rd Int. Cong. Rock Mechanics, Denver, vol. IIB 1974.

Panet M., Guénot A. - « Analysis of convergence behind the face of a tunnel $x$. Int Symp. Tunneling 82, Brighton, 1982 , p. $197-204$.

Ranken R.E., Ghaboussi J. - «Tunnel design consideration analysis of stress and deformations around advancing tunnels x. V.S. Department of transportation, Report FRA-OR\&D, 1975, p. 75-84.

Shahrour I. - «PECPLAS: a finite element software for the resolution of earthwork problems», Actes du colloque international Géotechnique et Informatique, Presses de l'ENPC. 1992, p. 327-334
Shahrour I. Ghorbanbeigi S. - Un programme d'étéments finis pour le calcul tridimensionnel des tunnels n. $6^{\circ} \mathrm{col}$ loque franco-polonais de Mécanique des sols appliquée, Douai, 20-22 septembre 1993, p. $256-263$

Shahrour 1., Ghorbanbeigi S. - "Calculation of turnels in soft ground $x$, Proceeding of the Third European Conference on Numerical Methods in Geotechnical Engineering, ECONMIG 94. Manchester, UK 1994. p. 229-234.

Swoboda G., Mertz W. Schmid A. "Three dimensional numerical models to simulate tunnel excavation s. Numerical Models in Geomechanics NUMOC III, Ed. Pietruszczak S. and Pande G.N. Elsevier Science Publishers Ltd, London, 1989 , p. 536-548,

Wittke W., Gell K. - $\alpha$ Three dimensional stability analyses for a shallow tunnel section of the subway of the city of Bochum 1, Construction Lot B3, Geotechnics, n³.1980, p. 111-119. 